\title{
Use of an endoscopic bioptome for extraction of a retained pacemaker lead tip
}

Sang Yong Ji, John Catanzaro, Jane E. Crosson, Jeffrey Brinker, Alan Cheng

Section of Cardiac Electrophysiology, Division of Cardiology, Johns Hopkins University School of Medicine, Baltimore, MD, U.S.A.

Submitted: 22 April 2012

Accepted: 16 July 2012

Arch Med Sci 2014; 10, 4: 853-854

DOI: 10.5114/aoms.2014.44962

Copyright $\odot 2014$ Termedia \& Banach

A 27-year-old male with D-transposition of the great arteries underwent a Mustard palliative procedure in infancy that was complicated by symptomatic bradycardia requiring implantation of an epicardial ventricular pacemaker at 1 year of age. His device was eventually upgraded to an endocardial dual chamber system when the epicardial lead failed to capture 8 years later. During this upgrade, an atrial lead (St Jude Medical, Tendril, $1688 \mathrm{TC}-52 \mathrm{~cm}$ ) was placed within the baffle and an endocardial ventricular lead (Guidant, Sweet Tip, 4269-52 cm) in the anatomic left ventricle (Figure 1). His lead thresholds over the years have progressively worsened requiring more frequent generator exchanges. He was subsequently referred for lead revision.

The ventricular lead was completely extracted with an LLD EZ® stylet and a $14 \mathrm{~F}$ excimer laser sheath (Spectranetics, Colorado Springs, CO). We then attempted to extract the atrial lead. The atrial lead was subsequently cut and extraction was attempted. Unfortunately, the lead fractured and unfurled, exposing a sharp end of the pacing cable within the vasculature. With the unfurled end difficult to visualize under fluoroscopy, this limited the ability of using existing tools for extraction (Figure 2). We subsequently employed the use of an endoscopic bioptome (Olympus FG-44 NR-1, reusable rat tooth alligator jaw rotatable grasping forcep) to successfully retrieve the distal tip of the lead (Figure 3).

The numbers of cardiac device implantations are steadily increasing [1] as well as the number of cases requiring extraction. Complete removal of the system is essential in those patients who have endovascular infection involving the lead with aggressive organisms such as Staphylococcus [2]. Therefore, there is great interest in improving this technique [3-5]. Currently available tools have their own advantages and limitations. As it was noted in our case, the limitation of the gooseneck snare is that it has to loop around the lead for successful retrieval but this was difficult to achieve given the orientation and state of the partially extracted lead (Figure 4). Encountering retained or remaining parts during an extraction case is not uncommon and often requires a more invasive approach to complete the extraction. This can not only result in fatal complications but also increase procedural morbidity. Here, we report of a challenging extraction case in which an endoscopic bioptome catheter was used to successfully retrieve the remaining part of an endocardial lead. As shown in Figure 3, precise localization and retrieval is feasible and relatively easier with the bioptome catheter.
Corresponding author:

Alan Cheng MD

$600 \mathrm{~N}$. Wolfe Street

Carnegie 568 Baltimore

MD 21287, U.S.A.

Phone: 4432872939

Fax: 4438735019

E-mail: alcheng@jhmi.edu 


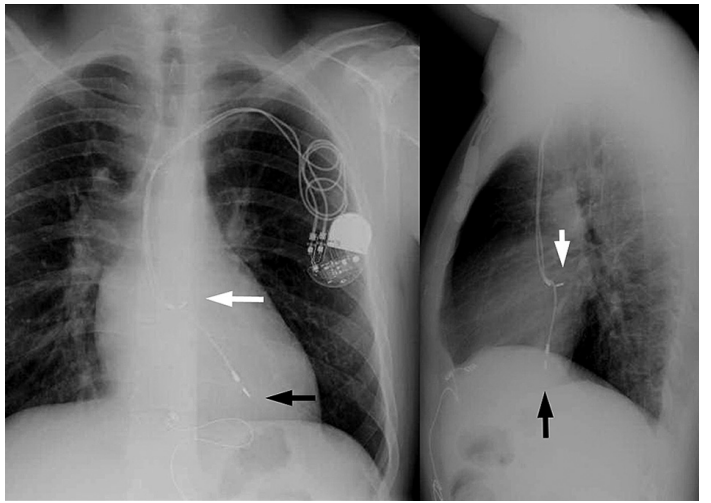

Figure 1. Posteroanterior and lateral chest $X$ rays that show an endocardial atrial lead (white arrow) placed within the baffle and an endocardial ventricular lead (black arrow) in the anatomic left ventricle

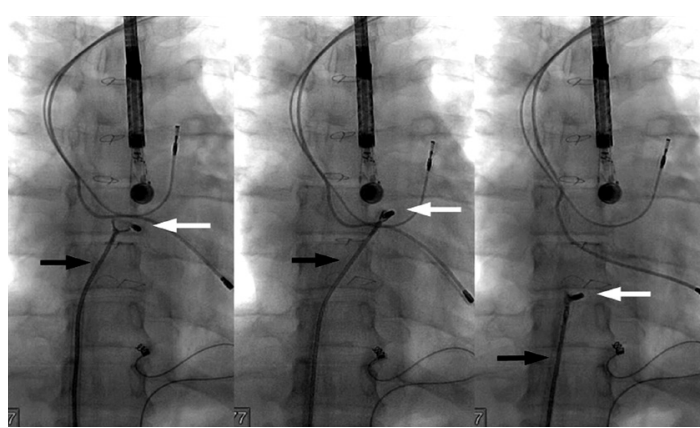

Figure 3. Fluoroscopic cine images that show retrieval of the remaining part of the old atrial lead (white arrow) with an endoscopic bioptome catheter (black arrow)

Challenging extraction cases in which parts of the leads are retained within the endocardial system, use of bioptome catheter under fluoroscopic guidance can facilitate complete removal of these parts.

\section{Reference}

1. Zhan C, Baine WB, Sedrakyan A, Steiner C. Cardiac device implantation in the United States from 1997 through 2004: a population-based analysis. J Gen Intern Med 2008; 23 (Suppl 1): 13-9.

2. Klug D, Lacroix D, Savoye C, et al. Systemic infection related to endocarditis on pacemaker leads. Clinical presentation and management. Circulation 1997; 95: 2098-107.

3. Jones IV SO, Eckart RE, Albert CM, Epstein LM. Large, single-center, single-operator experience with transvenous lead extraction: outcomes and changing indications. Heart Rhythm 2008; 5: 520-5.

4. Manolis AS, Maounis TN, Vassilikos V, Chiladakis J, Melita-Manolis H, Cokkinos DV. Ancillary tools in pacemaker and defibrillator lead extraction using a novel lead removal system. PACE 2001; 24: 282-7.

5. Lelakowski J, Kutarski A, Małecka B, Majewski J. Complex percutaneous extraction of a 15-year-old atrial lead dislodged into the subclavian vein. Arch Med Sci 2011; 7: 164-7.

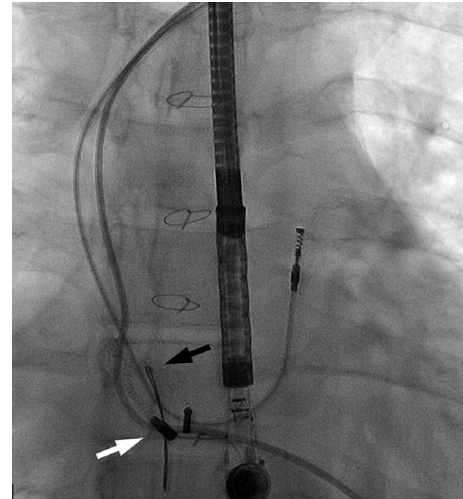

Figure 2. Fluoroscopic still image that shows the old broken atrial lead with its unfurled proximal portion (white arrow) and the gooseneck snare (black arrow)

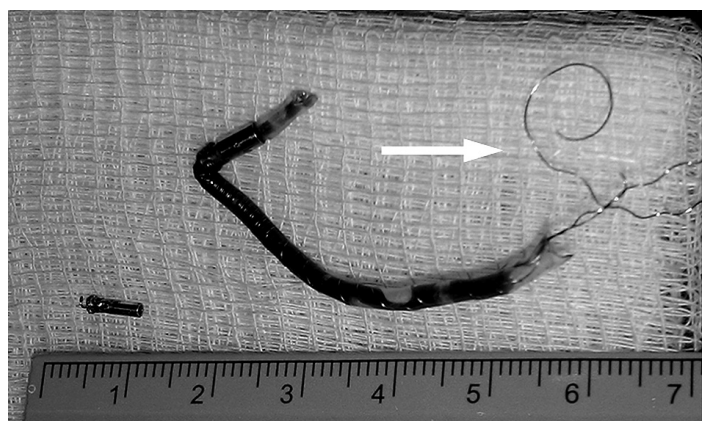

Figure 4. Retrieved portion of the old atrial lead that shows its unfurled proximal end (white arrow) 